\title{
Acoustic Studies of Molecular Interactions of Prasugrel-HCl Drug with Methanol at Various Temperatures
}

\author{
Lakshmi $\mathrm{D}^{1^{*}}$, Venkata Ramana $\mathrm{I}^{2}$ and Samatha $\mathrm{K}^{1}$ \\ ${ }^{1}$ Department of Physics, Andhra University, Visakhapatnam-530 003 \\ ${ }^{2}$ Department of Atomic Energy, Visakhapatnam-530 012 \\ ${ }^{1 *}$ dwarampudi.lakshmi@gmail.com
}

\begin{abstract}
Prasugrel hydrochloride drug is a Piperazine derivative which is used to prevent blood clotting. Molecular Interactions of Prasugrel hydrochloride with methanol liquid have been investigated using experimental and computational techniques. The drug has been added in specific quantities to $99 \%$ pure Methanol liquid to obtain certain molarity mixture solutions ranging from $0.025 \mathrm{M}$ to $0.2 \mathrm{M}$. The density, viscosity and ultrasonic velocity of the mixture solutions have been experimentally measured using standard techniques. From these values various parameters, such as Adiabatic Compressibility, Free Volume, Rao's constant, Relative Association, have been derived which show qualitatively the strength of intermolecular interactions between solute and solvent. Such studies have been done at various temperatures ranging from $303 \mathrm{~K}$ to $318 \mathrm{~K}$. It has been found that at low temperatures and low concentrations, there is a strong interaction between the solute and the solvent. It is due to H-bonding between solvent and solute molecules. At higher temperatures and low concentrations the interaction is relatively weak. But, at elevated temperatures and high concentrations, the strength of molecular interaction again increases.
\end{abstract}

Keywords: Prasugrel hydrochloride, Methanol, Ultrasonic Studies, Inter Molecular interactions, H-bonding.

\section{Introduction}

Understanding a drug's physico-chemical behaviour and intermolecular interaction with a solvent provide useful information for pharmaceutical and medicinal industry. The drug-solvent molecular interaction and its temperature dependence play an important role in the understanding of drug action [1,2]. Acoustical and Viscometric properties provide valuable clues for solute-solvent interactions in the solution mixture, which is helpful in predicting the absorption of drug and transport of drug across the biological membranes $[3,4]$. As there is demand from a local pharmaceutical company to study the interaction of Prasugrel- $\mathrm{HCl}$ with alcohols, we studied the drug's interaction with very simple alcohol which is Methanol.

Prasugrel is the generic name of the drug which is chemically designated as [5-[-2cyclopropyl-1-(2-fluorophenyl)-2-oxoethyl]-4,5,6,7-tetrahydrothieno[3,2-c]pyridin-2-yl] acetate. The trade or brand name of it is "Effient". The drug acts as a platelet inhibitor and is used to prevent thrombosis or blood clots in people with acute coronary syndrome who are undergoing a procedure after a recent heart attack or stroke, and in people with certain disorders of the heart or blood vessels. Prasugrel is a member of the thienopyridine class of ADP receptor inhibitors. Effient is formulated as the hydrochloride salt. It has the empirical formula $\mathrm{C}_{20} \mathrm{H}_{20} \mathrm{FNO}_{3} \mathrm{~S} . \mathrm{HCl}$ (or $\mathrm{C}_{20} \mathrm{H}_{21} \mathrm{ClFNO}_{3} \mathrm{~S}$ ) representing a molecular

${ }^{*}$ Corresponding Author 
weight of $409.9 \mathrm{~g} / \mathrm{mole}$. For completeness and forthcoming understanding of its interaction with the Methanol solvent, its molecular structure has been shown in Figure 1.<smiles>CC(=O)Oc1cc2c(s1)CCN(C(C(=O)C1CC1)c1ccccc1F)C2</smiles>

\section{Figure 1. Molecular Structure of Prasugrel-HCI}

In the present paper, we present the results obtained for experimental and computational study for understanding the intermolecular interactions of the Effient with Methanol solvent for different concentrations and at different temperatures. As the boiling point of the solvent is about $337 \mathrm{~K}$, we limited our study to the temperature range of $303 \mathrm{~K}$ to $318 \mathrm{~K}$. Experimental methods used for measuring density $(\rho)$, viscosity $(\eta)$ and ultrasonic velocity $(\mathrm{U})$ of the mixture solution have been presented in the next sections. Using these measured values, some quantities such as Adiabatic Compressibility, Free Volume, intermolecular free length, Rao's constant, Relative Association, etc., have been derived. From the nature of variation of these measured and derived parameters with concentration and temperature, the strength of interaction of the solute with the solvent has been deduced and explained.

\section{Experimental and Computational Techniques}

\section{Materials}

The drug Prasugrel hydrochloride (CAS No. 389574-19- 0, minimum assay value 98\%) has been used in this study. All the mixtures were prepared in Methanol on a morality basis by using Digital Balance with an accuracy of $\pm 0.1 \mathrm{mg}$. Methanol of HPLC grade (CAS No. 67-56- 1, minimum assay value of 99.9\%) procured from Avantor performancy materials India Ltd. has been used. The concentration of drug-methanol mixture has been varied in the range $0.025 \mathrm{M}$ to $0.2 \mathrm{M}$.

\section{Experimental Methods}

Standard experimental techniques have been used for measurement of density, viscosity and ultrasonic velocity in the mixture solution at different temperatures [5-14]. The density $(\rho)$ of the mixture solutions have been measured using a specific gravity bottle at temperatures $303.15 \mathrm{~K}, 308.15 \mathrm{~K}, 313.15 \mathrm{~K}, 318.15 \mathrm{~K}$. The accuracy in the density measurements is about $\pm 0.0001 \mathrm{~g} / \mathrm{ml}$. The viscometric measurements have been made by Ostwald's Viscometer with an accuracy of 0.001 N.s.m ${ }^{-2}$. The viscosity of the mixture solution has been obtained used the equation

$$
\eta=\eta_{s} \frac{\tau}{\tau_{s}} \frac{\rho}{\rho_{s}}
$$

Where $\eta, \rho$ and $\tau$ represent the viscosity, density and time of flow in Ostwald's viscometer. Quantities without subscript are for mixture solution and those with subscript 's' are for the solvent.

The ultrasonic velocity $(\mathrm{U})$ in the mixture has been measured by using variable path single crystal interferometer (Model F-81S, Mittal Enterprises, India) at a fixed frequency of $2 \mathrm{MHz}$ with an accuracy of $0.03 \%$. The temperature has been maintained with an 
accuracy of $0.1{ }^{\circ} \mathrm{C}$ by a water thermostat (Thermal Instruments $\backslash \&$ Equipments). For low temperature bath model, LTB-10 was used to circulate water through the double walled measuring cell made up of steel containing the experimental solution to attain the desired temperature. This device can be used for temperatures between $-10{ }^{\circ} \mathrm{C}$ to $90{ }^{\circ} \mathrm{C}$.

\section{Analytical/Computational Methods}

The measured values of $\eta, \rho$ and $U$ of the mixture solution can be used to derive certain quantities based on which the nature of the intermolecular interactions between the solute (Effient) and the solvent (Methanol) can be found out at a given concentration and temperature. The derived parameters are as listed below:

Adiabatic compressibility $(\chi)$ is a measure of the amount of compression that can be achieved in a material. Hence, it is also a good measure of how strong the intermolecular interactions are in the solution. $\chi$ of mixture solution can be computed from

$$
\chi=\frac{1}{\rho U^{2}}
$$

Intermolecular free length $\left(\mathrm{L}_{\mathrm{f}}\right)$ is the average distance between surfaces of neighbouring molecules. The variation of $\mathrm{L}_{\mathrm{f}}$ with the concentration of the solution and temperatures would reveal the strengthness of the intermolecular interactions. $\mathrm{L}_{\mathrm{f}}$ for a mixture can be obtained from

$$
L_{f}=K_{T} \chi^{1 / 2}
$$

where $\mathrm{K}_{\mathrm{T}}$ is a temperature dependent constant which is $205.8336 \times 10^{-8}$ (SI units).

Similarly, free volume $\left(\mathrm{V}_{\mathrm{f}}\right)$ is the volume in which an individual molecule moves in an average potential due to its neighbours. $\mathrm{V}_{\mathrm{f}}$ of a mixture solution can be obtained from

$$
\mathrm{V}_{\mathrm{f}}=\left(\frac{M_{e f f} U}{\eta K}\right)^{3 / 2}
$$

where $\mathrm{K}$ is constant equal to $4.28 \times 10^{9}$ (SI units). $\mathrm{M}_{\mathrm{eff}}=\Sigma \mathrm{m}_{\mathrm{i}} \mathrm{x}_{\mathrm{i}}$, where $\mathrm{m}_{\mathrm{i}}$ and $\mathrm{x}_{\mathrm{i}}$ are Molecular weight and mole fractions of the component ' $i$ '.

Acoustic impedance can be computed by

$$
Z=\rho U
$$

Relaxation time $(\tau)$ is the time taken for excitation energy to appear as translational energy and it depends on the temperature and impurities. This is also a measure of strength of intermolecular interaction. $\tau$ can be computed as

$$
\tau=\frac{4}{3} \eta \chi
$$

Molar Volume (V) of the solution can be calculated from

$$
V=\frac{M_{e f f}}{\rho}
$$

Vander Waals constant (b) can be calculated from 


$$
b=\frac{M_{e f f}}{\rho}\left[1-\left(\frac{R T}{M_{e f f} U^{2}}\right)\left\{\left(1+\frac{M_{e f f} U^{2}}{3 R T}\right)^{1 / 2}-1\right\}\right]
$$

where $\mathrm{R}$ is the gas constant $(=8.314 \mathrm{~J} / \mathrm{K} / \mathrm{mole})$ and $\mathrm{T}$ is the temperature.

Internal pressure ( $\Pi$ ) in the mixture is a cohesive force, which is a resultant of forces of attraction and forces of repulsion between molecules. Dissolved solutes exist under the internal pressure of medium and their interactions with the solvent, in general, arise through H-bonding, charge transfer, Columbic or Vanderwaal's interactions. Hence, $\Pi$ is also a measure of the strength of intermolecular interactions in a mixture solution and can be computed from

$$
\Pi=B R T\left(\frac{k \eta}{U}\right)^{1 / 2} \frac{\rho^{3 / 2}}{M_{e f f}^{7 / 6}}
$$

where $\mathrm{B}$ is the packing factor which is 2 .

Enthalpy of the medium can be given by

$$
H=\Pi V
$$

where $\mathrm{V}$ is the molar volume given by $\mathrm{M}_{\mathrm{eff}} / \rho$.

Rao's constant or Molar sound velocity can be computed from

$$
R=U^{1 / 3} V
$$

Wada's constant or Molar compressibility can be computed from

$$
W=\chi^{-1 / 7} V
$$

The relative association (RA) can be calculated from

$$
R A=\frac{\rho}{\rho_{s}}\left(\frac{U_{s}}{U}\right)^{1 / 3}
$$

We have developed a Fortran code called CIMIUS (Code for Intermolecular Interactions using Ultrasonic Studies). This software uses the measured values of density, viscosity and sound velocity at difference concentrations and temperatures and computes the above parameters and plots them.

\section{Results and Discussion}

Table 1 to 4 lists the measured and derived parameters using CIMIUS code for EffientMethanol mixtures for different concentrations of the solution and at different temperatures. 
Table 1. Measured Density, Sound Speed and Viscosity Values of EffientMethanol Solution at Different Concentrations and Temperatures

\begin{tabular}{|c|c|c|c|c|}
\hline $\begin{array}{c}\text { Temperature } \\
\text { (K) }\end{array}$ & $\begin{array}{c}\text { Concentration } \\
\text { (M) }\end{array}$ & $\begin{array}{l}\text { Density } \\
\left(\mathrm{kg} / \mathrm{m}^{3}\right)\end{array}$ & $\begin{array}{c}\text { Sound Speed } \\
(\mathrm{km} / \mathrm{s})\end{array}$ & $\begin{array}{c}\text { Viscosity } \\
\left.\text { (x } 10^{-3} \mathrm{~Pa}-\mathrm{sec}\right)\end{array}$ \\
\hline \multirow[t]{8}{*}{303} & 0.025 & 1566.64 & 1.089 & 1.1586 \\
\hline & 0.05 & 1582.61 & 1.09 & 1.2315 \\
\hline & 0.075 & 1592.39 & 1.096 & 1.321 \\
\hline & 0.1 & 1597.15 & 1.105 & 1.4071 \\
\hline & 0.125 & 1604.49 & 1.109 & 1.4549 \\
\hline & 0.15 & 1606.47 & 1.116 & 1.5083 \\
\hline & 0.175 & 1609.58 & 1.127 & 1.532 \\
\hline & 0.2 & 1607.69 & 1.141 & 1.6129 \\
\hline \multirow{8}{*}{308} & 0.025 & 1556.03 & 1.086 & 0.9806 \\
\hline & 0.05 & 1567.01 & 1.087 & 1.1085 \\
\hline & 0.075 & 1580.56 & 1.092 & 1.2096 \\
\hline & 0.1 & 1586.40 & 1.101 & 1.2140 \\
\hline & 0.125 & 1593.36 & 1.105 & 1.3833 \\
\hline & 0.15 & 1595.29 & 1.110 & 1.3747 \\
\hline & 0.175 & 1594.01 & 1.120 & 1.3941 \\
\hline & 0.2 & 1597.19 & 1.122 & 1.3456 \\
\hline \multirow[t]{8}{*}{313} & 0.025 & 1545.66 & 1.082 & 0.9343 \\
\hline & 0.05 & 1553.94 & 1.084 & 0.9893 \\
\hline & 0.075 & 1567.36 & 1.088 & 1.1491 \\
\hline & 0.1 & 1569.06 & 1.096 & 1.1200 \\
\hline & 0.125 & 1578.89 & 1.102 & 1.2895 \\
\hline & 0.15 & 1582.00 & 1.096 & 1.2921 \\
\hline & 0.175 & 1589.06 & 1.114 & 1.2978 \\
\hline & 0.2 & 1590.53 & 1.102 & 1.2990 \\
\hline \multirow[t]{8}{*}{318} & 0.025 & 1533.94 & 1.079 & 0.8779 \\
\hline & 0.05 & 1537.61 & 1.081 & 0.9493 \\
\hline & 0.075 & 1544.56 & 1.085 & 1.0628 \\
\hline & 0.1 & 1553.61 & 1.091 & 1.0591 \\
\hline & 0.125 & 1556.39 & 1.099 & 1.1210 \\
\hline & 0.15 & 1566.45 & 1.090 & 1.1383 \\
\hline & 0.175 & 1572.69 & 1.095 & 1.1530 \\
\hline & 0.2 & 1583.40 & 1.094 & 1.1812 \\
\hline
\end{tabular}

Table 2. Computed values of Compressibility, Impedance, Free-length and Free-Volume of Effient-Methanol Solution at different Concentrations and Temperatures

\begin{tabular}{|c|c|c|c|c|c|}
\hline $\begin{array}{l}\text { Tempe- } \\
\text { rature } \\
\text { (K) }\end{array}$ & $\begin{array}{c}\text { Concentration } \\
\text { (M) }\end{array}$ & $\begin{array}{c}\text { Compre- } \\
\text { ssibility } \\
\left(\mathbf{x} 10^{-9} \mathrm{~Pa}^{-1}\right)\end{array}$ & $\begin{array}{c}\text { Acoustic } \\
\text { Impedance } \\
\left(\mathbf{x} 10^{7} \mathrm{~kg} / \mathrm{m}^{2} / \mathrm{s}\right)\end{array}$ & $\begin{array}{c}\text { Free } \\
\text { Length } \\
\left(\begin{array}{cc}\left.\mathbf{x} 10^{-10} \mathrm{~m}\right)\end{array}\right.\end{array}$ & $\begin{array}{c}\text { Free } \\
\text { Volume } \\
\left(\begin{array}{ll}\mathbf{x} 10^{-8} \\
\left.\mathrm{~m}^{3} / \mathrm{mole}\right)\end{array}\right.\end{array}$ \\
\hline \multirow[t]{6}{*}{303} & 0.025 & .5382 & .1706 & .4775 & 1.883 \\
\hline & 0.05 & .5318 & .1725 & .4746 & 1.735 \\
\hline & 0.075 & .5227 & .1745 & .4706 & 1.589 \\
\hline & 0.1 & .5127 & .1764 & .4661 & 1.476 \\
\hline & 0.125 & .5067 & .1779 & .4633 & 1.423 \\
\hline & 0.15 & .4998 & .1792 & .4601 & 1.373 \\
\hline
\end{tabular}




\begin{tabular}{|c|c|c|c|c|c|}
\hline & 0.175 & .4891 & .1813 & .4552 & 1.373 \\
\hline & 0.2 & .4772 & .1835 & .4496 & 1.307 \\
\hline \multirow[t]{8}{*}{308} & 0.025 & .5449 & .1689 & .4804 & 2.408 \\
\hline & 0.05 & .5400 & .1703 & .4783 & 2.024 \\
\hline & 0.075 & .5305 & .1725 & .4741 & 1.803 \\
\hline & 0.1 & .5200 & .1746 & .4693 & 1.831 \\
\hline & 0.125 & .5139 & .1760 & .4666 & 1.527 \\
\hline & 0.15 & .5087 & .1770 & .4642 & 1.565 \\
\hline & 0.175 & .5001 & .1785 & .4603 & 1.567 \\
\hline & 0.2 & .4973 & 1792 & .4590 & 1.671 \\
\hline \multirow{8}{*}{313} & 0.025 & .5526 & .1672 & 4838 & 2.575 \\
\hline & 0.05 & .5476 & .1684 & .4816 & 2.390 \\
\hline & 0.075 & .5389 & .1705 & .4778 & 1.937 \\
\hline & 0.1 & .5305 & .1719 & .4741 & 2.053 \\
\hline & 0.125 & .5215 & .1739 & .4700 & 1.690 \\
\hline & 0.15 & .5262 & .1733 & .4721 & 1.685 \\
\hline & 0.175 & .5070 & . 1770 & .4635 & 1.730 \\
\hline & 0.2 & .5177 & .1752 & .4683 & 1.715 \\
\hline \multirow[t]{8}{*}{318} & 0.025 & .5599 & .1655 & .4870 & 2.815 \\
\hline & 0.05 & .5565 & .1662 & .4855 & 2.533 \\
\hline & 0.075 & .5499 & .1675 & .4827 & 2.169 \\
\hline & 0.1 & .5407 & .1694 & .4786 & 2.217 \\
\hline & 0.125 & .5319 & .1710 & .4747 & 2.076 \\
\hline & 0.15 & .5373 & .1707 & .4771 & 2.021 \\
\hline & 0.175 & .5303 & .1722 & .4740 & 2.014 \\
\hline & 0.2 & .5276 & .1732 & .4728 & 1.956 \\
\hline
\end{tabular}

Table 3. Computed Values of Relative Association, Viscous Relaxation Time, Molar Volume and Internal Pressure of Effient-Methanol Solution at Different Concentrations and Temperatures

\begin{tabular}{|c|c|c|c|c|c|}
\hline $\begin{array}{c}\text { Tempe- } \\
\text { rature } \\
(\mathbf{K})\end{array}$ & $\begin{array}{c}\text { Concentration } \\
(\mathbf{M})\end{array}$ & $\begin{array}{c}\text { Relative } \\
\text { Association }\end{array}$ & $\begin{array}{c}\text { Relaxation } \\
\text { Time } \\
\left(\mathbf{x ~ 1 0} \mathbf{1 0}^{-12} \mathbf{s}\right)\end{array}$ & $\begin{array}{c}\text { Molar } \\
\text { Volume } \\
(\mathbf{l t} / \mathbf{m o l e})\end{array}$ & $\begin{array}{c}\text { Internal } \\
\text { Pressure } \\
(\mathbf{G P a})\end{array}$ \\
\hline \multirow{3}{303}{} & 0.025 & 1.986 & .8315 & .02057 & .3833 \\
\cline { 2 - 6 } & 0.05 & 2.006 & .8732 & .02048 & .4038 \\
\cline { 2 - 6 } & 0.075 & 2.014 & .9208 & .02047 & .4238 \\
\cline { 2 - 6 } & 0.1 & 2.015 & .9621 & .02053 & .4405 \\
\cline { 2 - 6 } & 0.125 & 2.022 & .9830 & .02055 & .4532 \\
\cline { 2 - 6 } & 0.15 & 2.020 & .1005 & .02065 & .4640 \\
\cline { 2 - 6 } & 0.175 & 2.017 & .9991 & .02072 & .4697 \\
\hline \multirow{4}{*338}{} & 0.2 & 2.006 & .1026 & .02087 & .4812 \\
\cline { 2 - 6 } & 0.025 & 1.974 & .7125 & .02071 & .3553 \\
\cline { 2 - 6 } & 0.05 & 1.988 & .7982 & .02068 & .3842 \\
\cline { 2 - 6 } & 0.075 & 2.002 & .8557 & .02063 & .4084 \\
\cline { 2 - 6 } & 0.1 & 2.004 & .8417 & .02067 & .4125 \\
\cline { 2 - 6 } & 0.125 & 2.010 & .9480 & .02070 & .4454 \\
\cline { 2 - 6 } & 0.15 & 2.010 & .9325 & .02079 & .4468 \\
\cline { 2 - 6 } & 0.175 & 2.002 & .9296 & .02093 & .4503 \\
\cline { 2 - 6 } & 0.2 & 2.005 & .8923 & .02100 & .4463 \\
\hline
\end{tabular}




\begin{tabular}{|c|c|c|c|c|c|}
\hline \multirow[t]{8}{*}{313} & 0.025 & 1.964 & .6884 & .02085 & .3496 \\
\hline & 0.05 & 1.973 & .7224 & .02086 & .3647 \\
\hline & 0.075 & 1.988 & .8257 & .02080 & .4002 \\
\hline & 0.1 & 1.985 & .7923 & .02090 & .3969 \\
\hline & 0.125 & 1.994 & .8967 & .02089 & .4316 \\
\hline & 0.15 & 2.001 & .9065 & 02097 & .4374 \\
\hline & 0.175 & 1.999 & .8775 & .02099 & .4407 \\
\hline & 0.2 & 2.008 & .8967 & .02109 & .4468 \\
\hline \multirow[t]{8}{*}{318} & 0.025 & 1.951 & 6555 & 02101 & 3408 \\
\hline & 0.05 & 1.954 & .7044 & .02108 & .3578 \\
\hline & 0.075 & 1.960 & .7793 & .02111 & .3830 \\
\hline & 0.1 & 1.968 & .7636 & .02111 & .3872 \\
\hline & 0.125 & 1.967 & .7951 & .02119 & .4007 \\
\hline & 0.15 & 1.985 & .8155 & .02117 & .4121 \\
\hline & 0.175 & 1.990 & .8152 & .02121 & .4191 \\
\hline & 0.2 & 2.004 & .8310 & .02119 & .4315 \\
\hline
\end{tabular}

Table 4. Computed values of Rao's Constant, Wada's Constant, Vander Waals Constant and Enthalpy of Effient-Methanol Solution at different Concentrations and Temperatures

\begin{tabular}{|c|c|c|c|c|c|}
\hline $\begin{array}{l}\text { Tempe } \\
\text {-rature } \\
\text { (K) }\end{array}$ & $\begin{array}{l}\text { Concen- } \\
\text { tration (M) }\end{array}$ & $\begin{array}{l}\text { Rao's constant } \\
(\mathrm{lt} / \mathrm{mole})(\mathrm{m} / \mathrm{s})^{1 / 3}\end{array}$ & $\begin{array}{c}\text { Wada } \\
\text { constant } \\
(1 \mathrm{t} / \mathrm{mole}) \mathbf{P a}^{1 / 7}\end{array}$ & $\begin{array}{l}\text { Van der } \\
\text { Waal } \\
\text { constant } \\
\text { (lt/mole) }\end{array}$ & $\begin{array}{c}\text { Enthalpy } \\
\left(\begin{array}{c}\text { (x 10 } \\
\text { J/mole) }\end{array}\right.\end{array}$ \\
\hline \multirow[t]{8}{*}{303} & 0.025 & .2116 & .4339 & .01859 & 7.886 \\
\hline & 0.05 & .2108 & .4328 & .01851 & 8.272 \\
\hline & 0.075 & .2111 & .4337 & .01852 & 8.679 \\
\hline & 0.1 & .2123 & .4361 & .01858 & 9.046 \\
\hline & 0.125 & .2128 & .4374 & .01861 & 9.318 \\
\hline & 0.15 & .2142 & .4402 & .01870 & 9.582 \\
\hline & 0.175 & .2157 & .4432 & .01879 & 9.738 \\
\hline & 0.2 & .2181 & .4478 & .01894 & 10.04 \\
\hline \multirow[t]{8}{*}{308} & 0.025 & .2129 & .4361 & .01870 & 7.360 \\
\hline & 0.05 & .2127 & .4361 & .01868 & 7.949 \\
\hline & 0.075 & .2124 & .4360 & .01864 & 8.426 \\
\hline & 0.1 & .2134 & .4382 & .01869 & 8.528 \\
\hline & 0.125 & .2140 & .4395 & .01873 & 9.221 \\
\hline & 0.15 & .2153 & .4422 & .01882 & 9.291 \\
\hline & 0.175 & .2173 & .4461 & .01895 & 9.426 \\
\hline & 0.2 & .2182 & .4481 & .01903 & 9.375 \\
\hline \multirow{8}{*}{313} & 0.025 & .2140 & .4381 & .01881 & 7.290 \\
\hline & 0.05 & .2143 & .4389 & .01883 & 7.610 \\
\hline & 0.075 & .2139 & .4387 & .01878 & 8.326 \\
\hline & 0.1 & .2155 & .4418 & .01888 & 8.298 \\
\hline & 0.125 & .2157 & .4426 & .01888 & 9.018 \\
\hline & 0.15 & .2162 & .4437 & .01895 & 9.174 \\
\hline & 0.175 & .2176 & .4466 & .01900 & 9.253 \\
\hline & 0.2 & .2179 & .4474 & .01908 & 9.426 \\
\hline
\end{tabular}




\begin{tabular}{|c|c|c|c|c|c|}
\hline 318 & 0.025 & .2155 & .4407 & .01894 & 7.162 \\
\cline { 2 - 6 } & 0.05 & .2163 & .4426 & .01901 & 7.544 \\
\cline { 2 - 6 } & 0.075 & .2169 & .4439 & .01905 & 8.087 \\
\cline { 2 - 6 } & 0.1 & .2173 & .4449 & .01906 & 8.176 \\
\cline { 2 - 6 } & 0.125 & .2187 & .4478 & .01914 & 8.493 \\
\cline { 2 - 6 } & 0.15 & .2179 & .4468 & .01912 & 8.729 \\
\cline { 2 - 6 } & 0.175 & .2186 & .4484 & .01917 & 8.891 \\
\cline { 2 - 6 } & 0.2 & .2183 & .4482 & .01915 & 9.145 \\
\hline
\end{tabular}

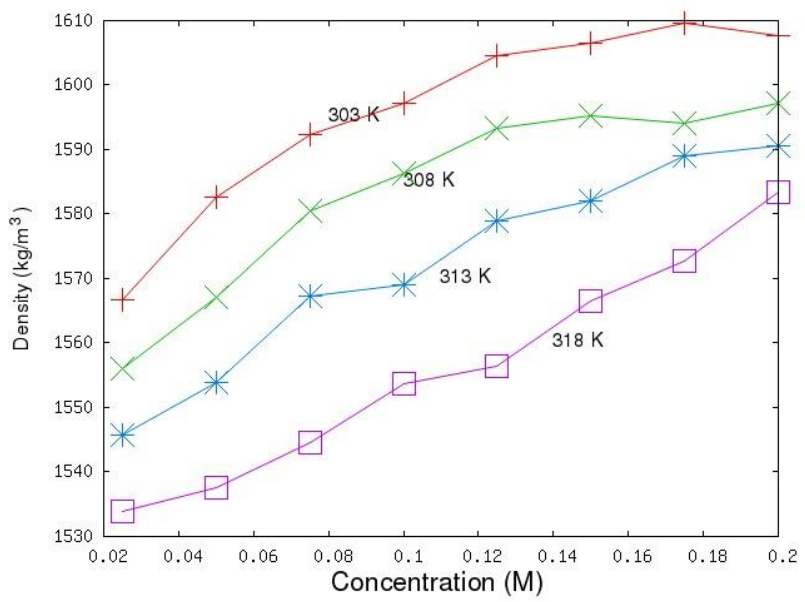

Figure 2. Measured Density of Effient-Methanol Solution at Different Concentrations and Temperatures

Figure 2 shows the variation of density of the drug solution with concentration at different temperatures. The density of the solution increases almost linearly with concentration. Since, the volumes of the solution are equal in all cases; the increase in the density indicates increasing number of molecules per unit volume. Hence, this indicates strong interaction between solute-solvent molecules. As the temperature increases, the density decreases as a result of increase in various bond lengths.

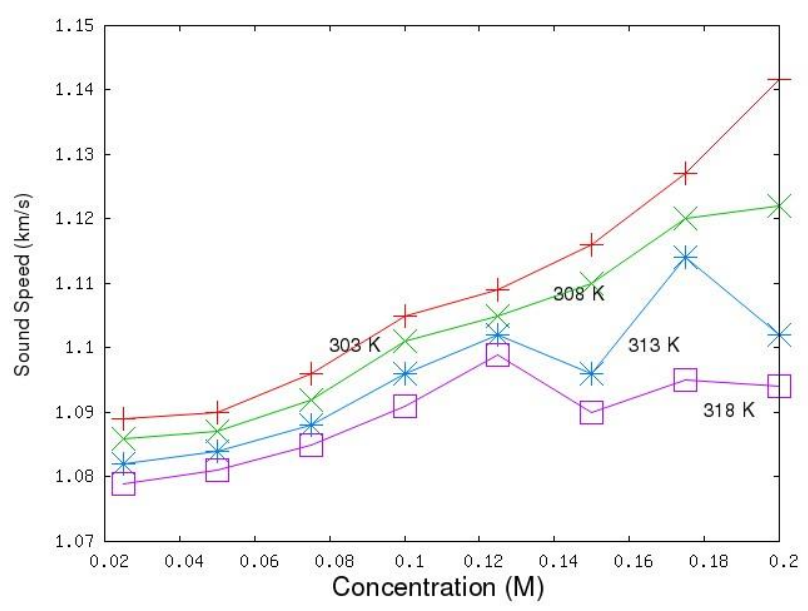

Figure 3. Measured Sound Speed in Effient-Methanol Solution at Different Concentrations and Temperatures

Figure 3 shows the variation of the sound speed in the solution with concentration and temperature. In general, the sound speed has been observed to be increasing with the concentration, especially at low temperatures $(<310 \mathrm{~K})$. This is consistent with the 
density variation and indicates strong intermolecular interactions between solute-solvent molecules. For a given concentration, as the temperature of the solution increases, the sound speed slightly decreases, which is also due to decrease in density at elevated temperatures. At high temperatures and high concentrations, the sound speed is observed to be attaining a saturated value.

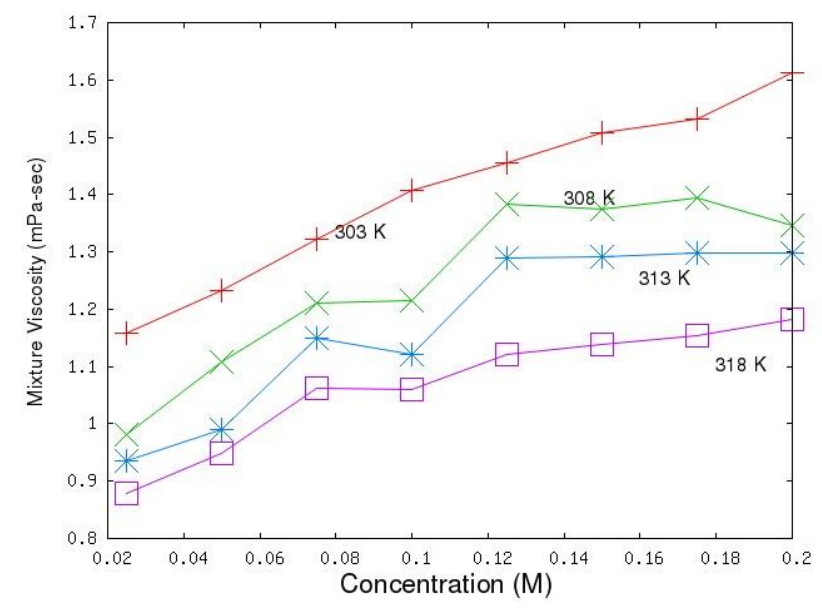

\section{Figure 4. Measured Viscosity of Effient-Methanol Solution at Different Concentrations and Temperatures}

The variation of viscosity of the Effient-methanol solution with concentration and temperature has been shown in the Figure 4. It can be seen that the viscosity of the mixture solution increases with concentration in general. Viscosity is due to cohesive or shear forces between different molecules or layers in the solution. Hence, increase in the viscosity means enhancement of the cohesive forces between solute-solvent-solvent molecules, which again indicate the existence of strong intermolecular forces between solute-solvent molecules. At a given concentration, the viscosity decreases with increase in temperature, which is consistent with the density variation.

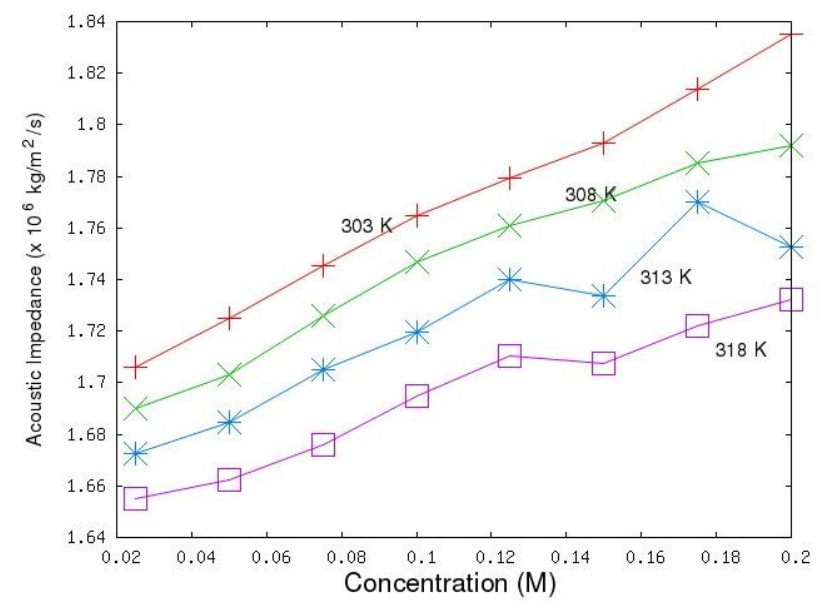

Figure 5. Calculated Impedance of Effient-Methanol Solution at Different Concentrations and Temperatures

Acoustic impedance $(Z)$ is a measure of resistance of the medium to introduction of disturbances. This is equal to the product of density and sound speed of the medium. The amount of sound wave transmission or reflection at interfaces depends on the acoustic impedance of the medium. Hence, $\mathrm{Z}$ is an important parameter to be studied. Figure 5 
shows the variation of $\mathrm{Z}$ with concentration and temperature of the solution. $\mathrm{Z}$ increases with the concentration at a given temperature and decreases with temperatures at a given concentration. These trends are consistent with the density and sound speed variations.

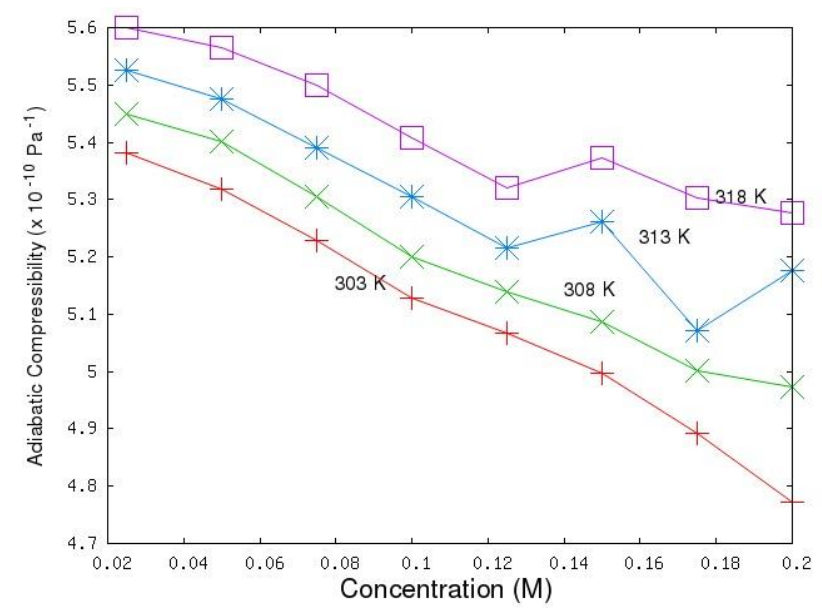

Figure 6. Calculated Adiabatic Compressibility of Effient-Methanol Solution at Different Concentrations and Temperatures

Adiabatic compressibility $(\chi)$ is a measure of change in volume of the medium for applied pressure or forces. This is reciprocal of bulk modulus. Figure 6 shows the variation of the adiabatic compressibility with concentration and temperature. $\chi$ decreases as the concentration increases at a given temperature. It is due to the fact that the density increases with the concentration and as the density increases it becomes increasingly difficult to compress a medium. Hence, these variations in $\chi$ show the existence of strong solvent-solute interactions. At a given concentration, the compressibility increases with the temperature. It is due to the fact that as the temperature increases, the bond lengths increase and there is greater scope for further compression of the medium.

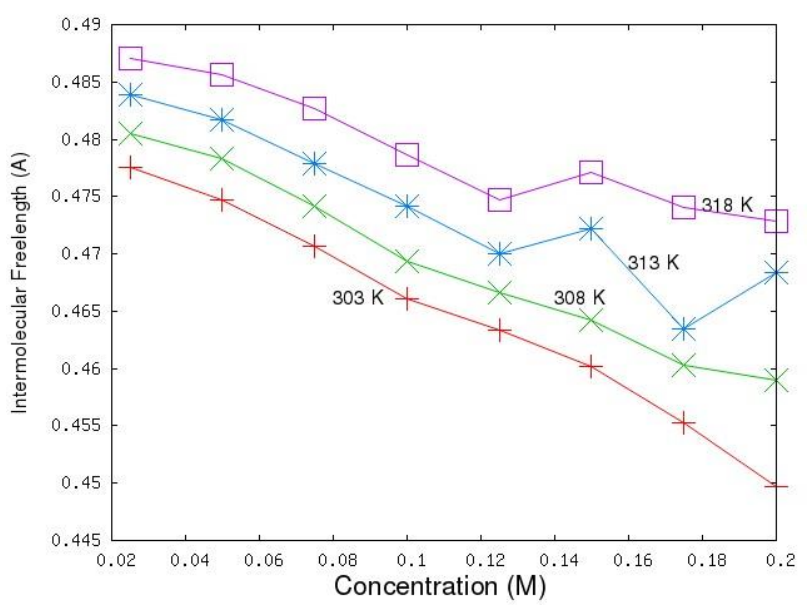

Figure 7. Calculated Free-length in Effient-Methanol Solution at Different Concentrations and Temperatures

Figure 7 shows the variation of intermolecular free length of the mixture solution with concentration and temperature. At a given temperature, as the concentration increases, $\mathrm{L}_{\mathrm{f}}$ decreases. It means the average distance between surfaces of neighbouring molecules is getting reduced. This indicates that the attractive forces between the molecules have become strong than in the pure solvent, which indicate strong solute-solvent interactions. 
At high temperatures and high concentrations, the data is noisy and indicate weak interaction. At a given concentration, the free length increases with increase in temperature which is as expected.

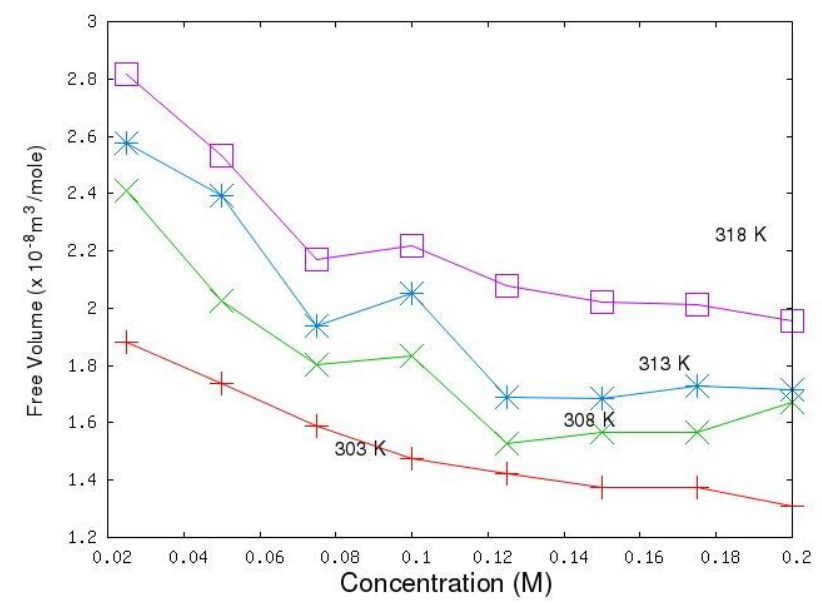

Figure 8. Calculated Free Volume in Effient-Methanol Solution at Different Concentrations and Temperatures

Similar trends as of free-length have been observed in the variation of Free Volume $\left(\mathrm{V}_{\mathrm{f}}\right)$ with concentration and temperature as shown in the Figure 8.

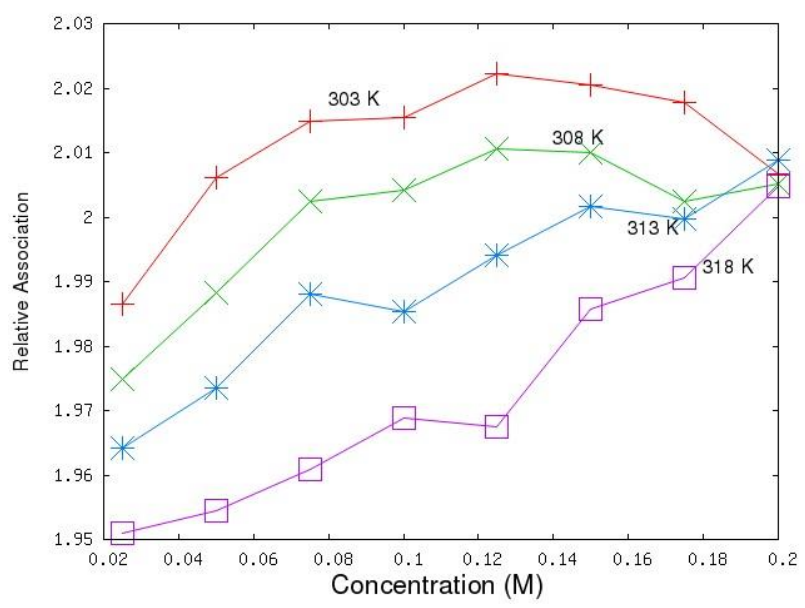

Figure 9. Calculated Relative Association in Effient-Methanol Solution at Different Concentrations and Temperatures

Figure 9 shows the variation of Relative Association (RA) of the mixture solution with concentration and temperature. At low temperatures, relative association increases with the concentration and saturates to a value of about 2.01 at higher concentration. At higher temperatures, there is steady increase in the RA with concentration. Such increase in the RA indicates strong solute-solvent interaction and saturation in the curve indicate saturation of solute-solvent mixture. For a given concentration, the RA decreases with increase in temperature, which is due to increase in bond lengths and lattice vibrations at elevated temperatures. 


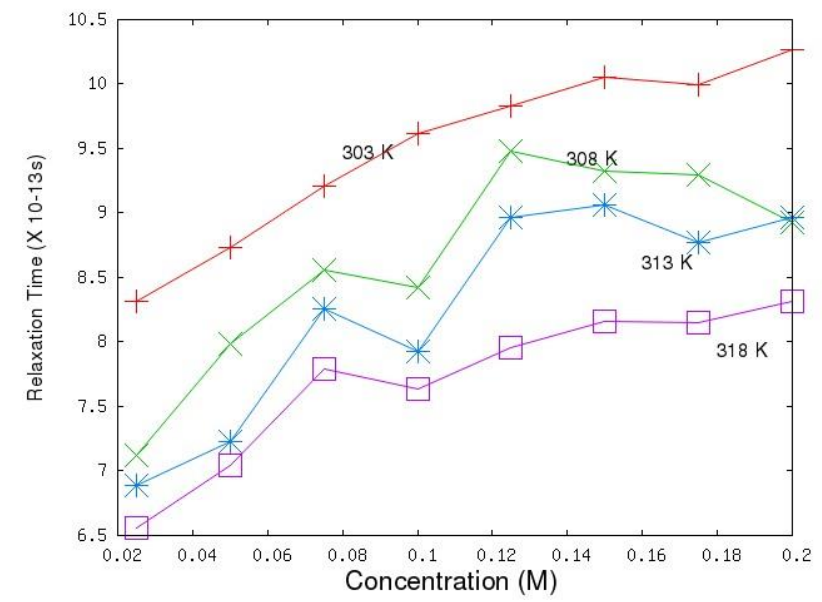

Figure 10. Calculated Relaxation Time in Effient-Methanol Solution at Different Concentrations and Temperatures

Figure 10 shows the variation of viscous relaxation time $(\tau)$ in the mixture solution with concentration and temperature. As the concentration increases, in general, $\tau$ increases. That means, any external excitation energy given to the solution, would take increasingly more time to convert into translational degrees of freedom or translational energy as the concentration increases. This increase in $\tau$ is due to increase in viscosity of the solution with concentration. At higher temperatures and high concentrations, the change in $\tau$ is not as marginal as at low concentrations. This again follows the viscosity variation trends shown in the Figure 4. At a given concentration, as the temperature is increased, $\tau$ decreases. This is due to increase in the lattice vibrations at higher temperatures, which would help in easier dissipation of excitation energy into translational energy.

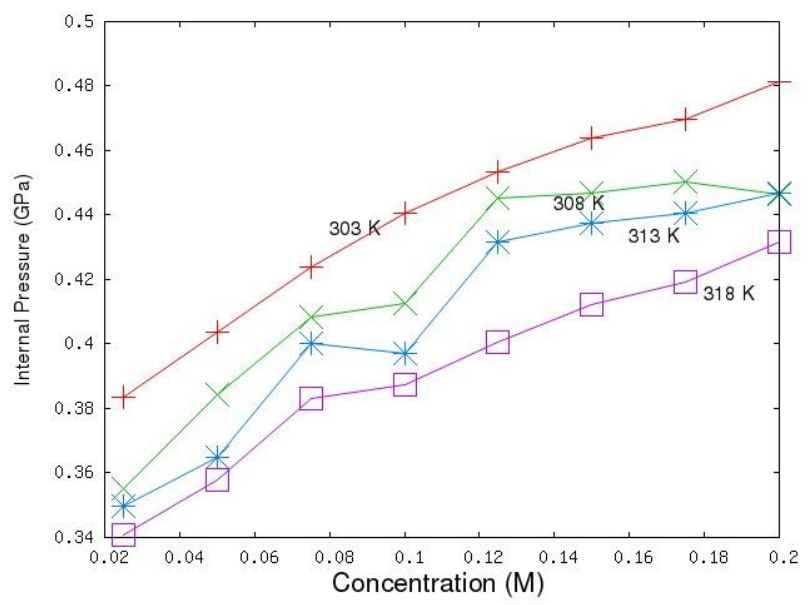

Figure 11. Calculated Internal Pressure in Effient-Methanol Solution at Different Concentrations and Temperatures

The variation of internal pressure in the Effient-methanol mixture with concentration and temperature has been shown in the Figure 11. Internal pressure or stress is due to the internal arrangement of the molecules. As the molecular arrangement changes with the concentration, rearrangement of molecules would take place, which would alter the internal stress field or internal pressure. It has been observed from the Figure 11 that as the concentration increases the internal pressure increases almost linearly. This would 
indicate stronger solute-solvent interactions in the solution. Similarly, enthalpy of the mixture solution increases with concentration as shown in the Figure 12.

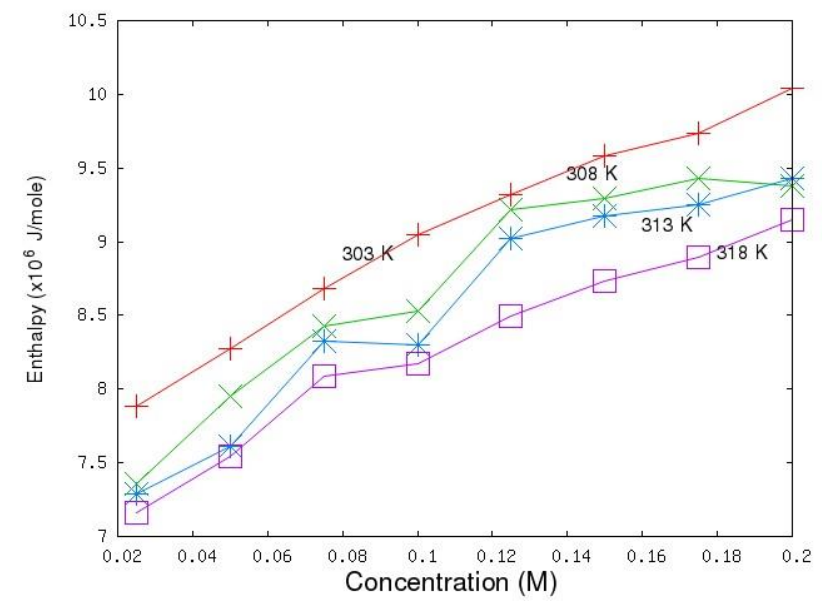

Figure 12. Calculated Enthalpy in Effient-Methanol Solution at Different Concentrations and Temperatures

Figures 13-16 show variation of molar volume, Vander Waal constant, Rao's constant and Wada constant respectively of the Effient-methanol solution with concentration and temperature. The trends of variation of these quantities appear almost the same. At low temperatures the variation with concentration is non-linear and at high temperatures it is almost linear resembling that of ideal solution mixture. At low concentrations and low temperatures there is negative deviation from ideal behaviour. As the concentration further increases the variation in the quantity becomes linear. Such nonlinear behaviour indicates strong long-range interaction between solute and solvent molecules.

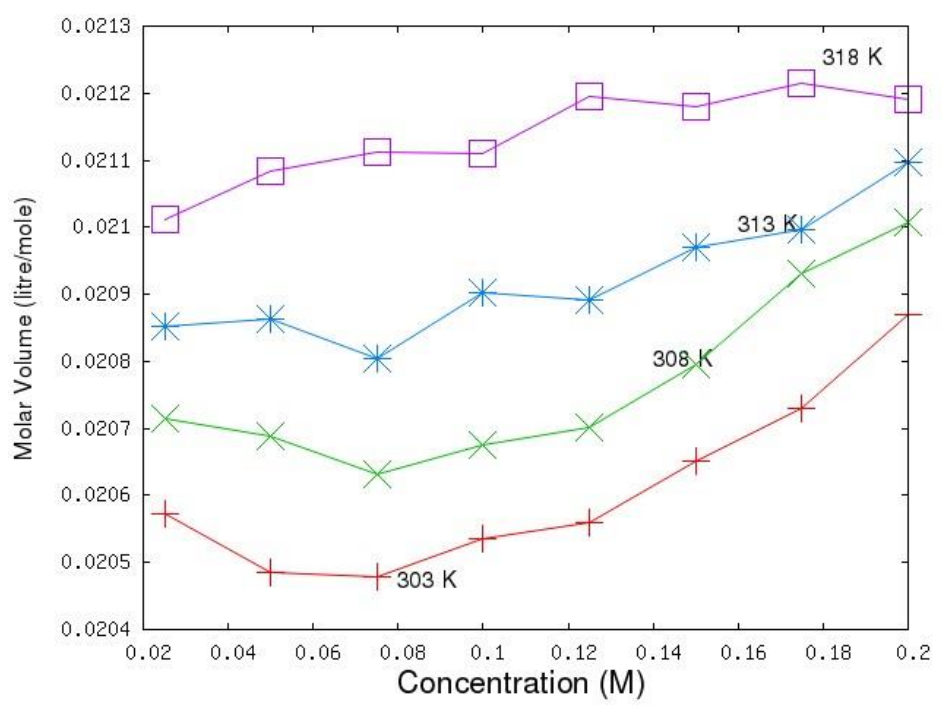

Figure 13. Calculated Molar Volume of Effient-Methanol Solution at Different Concentrations and Temperatures 


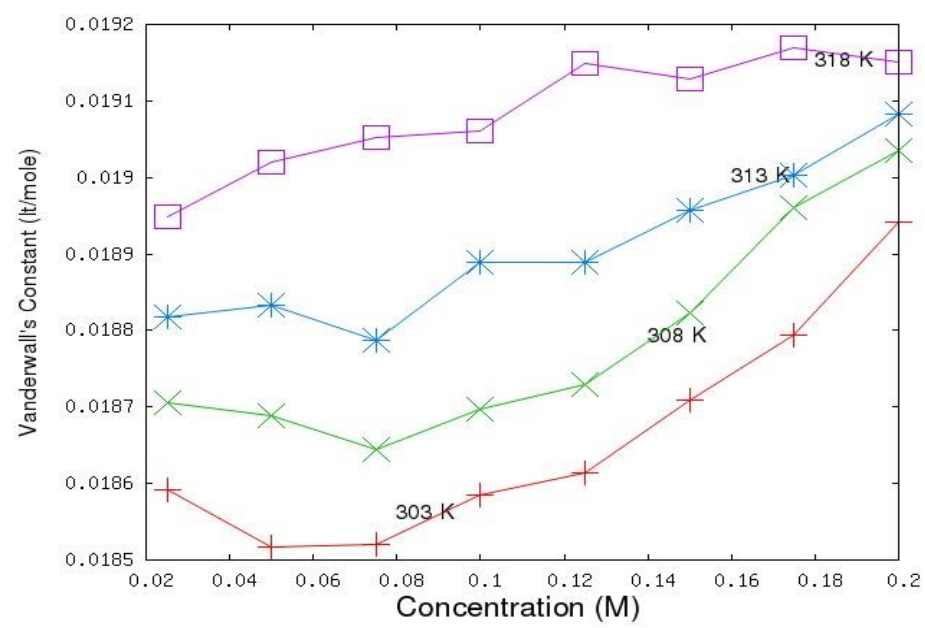

Figure 14. Calculated van der Waal's Constant of Effient-Methanol Solution at Different Concentrations and Temperatures

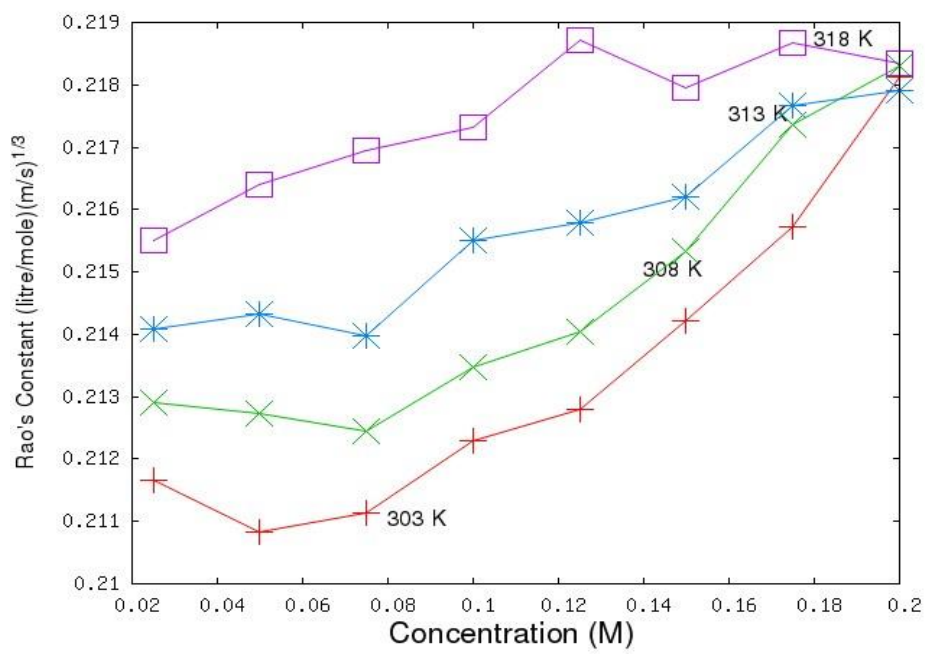

Figure 15. Calculated Rao's Constant of Effient-Methanol Solution at Different Concentrations and Temperatures

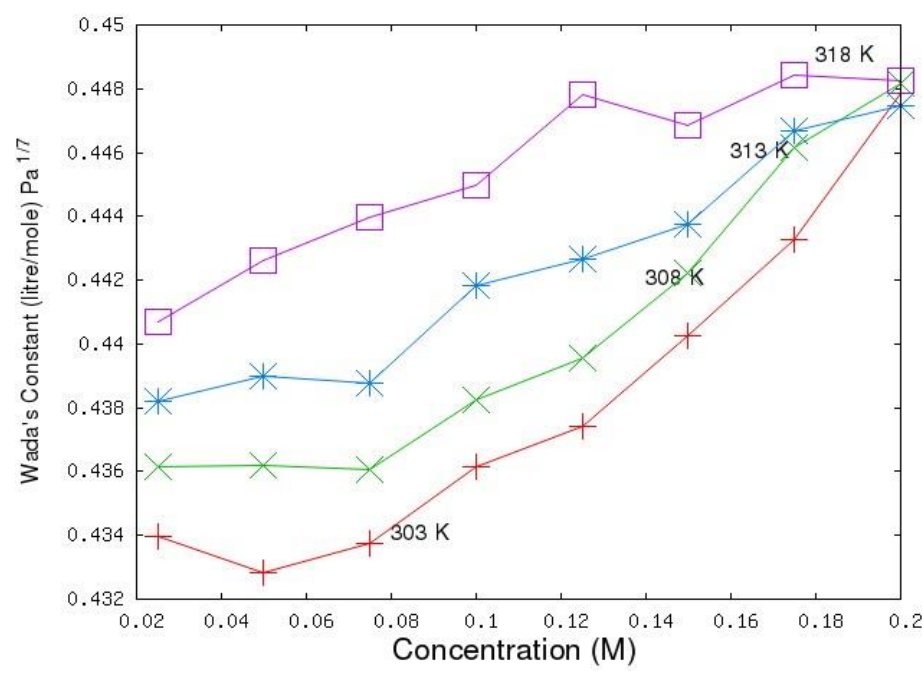

Figure 16. Calculated Wada's Constant of Effient-Methanol Solution at Different Concentrations and Temperatures 


\section{Nature of Solute-Solvent interaction:}

In view of the above results and discussion, it can be concluded that there exists strong solute-solvent interaction. Especially the nonlinear behaviour of molar volume, Rao's constant, etc. strongly indicate this point. The reason for such strong interaction between Effient and methanol is due to formation of $\mathrm{H}$-bonds between those molecules, which can be explained using their molecular structures.

Hydrogen bonding is a very strong type of dipole-dipole attraction, which is due to electrostatic attraction between two polar groups that occurs when an $\mathrm{H}$ atom covalently bound to a highly electronegative atom such as $\mathrm{N}, \mathrm{O}$, or $\mathrm{F}$ experiences the electrostatic field of another highly electronegative atom nearby. Hydrogen bonds exist between different molecules (intermolecular) of methanol.

The molecular structure of Effient has already been shown in the Figure 1. From reference [15], it can be found out that the Hydrogen Bond Donor Count and Acceptor Count for an Effient molecule are 1 and 6 respectively. That means, an Effient molecule has one donor atoms for $\mathrm{H}$ bonding with other molecules and six acceptor atoms for $\mathrm{H}$ bonding from other molecules. Hence, when Effient is added to methanol, strong H-bonds will form between solute and solvent molecules. This is why we see indication of existence strong solute-solvent interactions in all the figures and results discussed above.

\section{Conclusions}

Molecular Interactions of Prasugrel hydrochloride with methanol liquid have been investigated using experimental and computational techniques. The drug has been added in specific quantities to $99 \%$ pure Methanol liquid to obtain certain morality mixture solutions ranging from $0.025 \mathrm{M}$ to $0.2 \mathrm{M}$. The density, viscosity and ultrasonic velocity of the mixture solutions have been experimentally measured using standard techniques. Indigenously developed CIMIUS code has been used to compute various parameters, such as Adiabatic Compressibility, Free Volume, Rao's constant, Relative Association using the above experimentally obtained values. Such studies have been done at various temperatures ranging from $303 \mathrm{~K}$ to $318 \mathrm{~K}$. It has been found that at low temperatures and low concentrations, there is a strong interaction between Effient and Methanol. It is due to $\mathrm{H}$-bonding between solvent and solute molecules. At higher temperatures and low concentrations the interaction is relatively weak. But, at elevated temperatures and high concentrations, the strength of molecular interaction again increases.

\section{Acknowledgments}

One of the authors, Lakshmi D., would like to acknowledge the University Grants Commission, India for the award of Teacher Fellowship to perform current research work in Department of Physics, Andhra University.

\section{References}

[1] S. S. Dhondge, S. P. Zodape and D. V. Parwate, "Volumetric and viscometric studies of some drugs in aqueous solutions at different temperatures", J. Chem. Thermodynamics, vol. 48, (2012), pp. 207-212.

[2] S. K. Thakur and S. Chauhan, "Ultrasonic velocity and allied parameters of drug colimax in aq. 1propanol at 298.15K", J. Chem. Pharm. Research, vol. 3, no. 2, (2011), pp. 657-664.

[3] S. S. Dhondge, R. L. Paliwal, N. S. Bhave and C. P. Pandhurnekar, "Study of thermodynamic properties of aq. binary mixture of glycine, I-alanine and $\beta$-alanine at low temp", $(\mathrm{T}=275.15,279.15$, and 283.15) K”, J. Chem Thermodynamics, vol. 45, (2012), pp. 114-121.

[4] V. K. Syal, A. Chauhan and S. Chauhan, "Ultrasonic velocity, viscosity and density study of (PEG 8,000, PEG - 20,000) in acetonitrile $\mathrm{H}_{2} \mathrm{O}$ mixture at 250 C", J. Pure Appl. Ultrasonics, vol. 27, (2005), pp. 61-69.

[5] Sh. Baluja, A. Solanki and N. Kachhadia, "An ultrasonic study of some drugs in solution", Russian J. Phy. Chem. A, vol. 81, no. 5, (2007), pp. 742-746. 
[6] A. N. Sonar and N. S. Pawar, "Ultrasonic velocity, density \&amp; viscosity measurement of substituted heterocyclic drugs in 1,4-dioxane at 303 K", Rasayan J. Chem., vol. 3, no. 1, (2010), pp. 38-43.

[7] I. Dalmazio, M. A. Tania, Alves and R. Augusti, "An Appraisal on the Degradation of Paracetamol by $\mathrm{TiO}_{2} /$ UV System in Aq. Medium Product Identn by GC-MS”, J. Braz. Chem. Society, vol. 19, no. 1, (2008), pp. 81-88.

[8] Silva, R. Joseane, Queiroz, M. D. Luana, N. Auriekson, Souza, J. C. Pergentino, Borges and S. Rosivaldo, "A Theoretical study of Paracetamol Acyl-Ether Derivatives", J. Comp. and Theoretical Nanoscience, vol. 8(4), (2011), pp. 670-675.

[9] A. D. Nikam, S. P. Sampada and S. V. Gandhi, "Estimation of paracetamol and aceclofenac in tablet formulation by ratio spectra derivative Spectroscopy", Ind. J. Pharma. Sciences, vol. 70(5), (2008), pp. 635-637.

[10] van de Straat R., G. J. Biilooand N. P. Vermeulen, "Paracetamol, 3-monoalkyl- and 3,5-dialkylsubstances derived Antioxidant activity and relationship between lipid peroxidation and cytotoxicity", Biochem. Pharmacol. Vol. 37(18), (1988), pp. 3473-3476.

[11] A. Tadkalkar, K. J. Deshpande and G. K. Bichile, "Ultrasonic study on interaction of inorganic salts with fructose", Scholars Res. Library, vol. 2(4), (2011), pp. 80-88.

[12] M. Thirunavukkarasu and N. Kanagathara, "Ultrasonic study on Non-Aq. Solution of Toluene in $\mathrm{CCl}_{4}$ ", Int. J. ChemTech. Research, vol. 4(1), (2012), pp. 459-463.

[13] C. Roumana and G. Velraj, "Molecular interaction study of antibiotic Doxycycline hyclate with linoleic acid", Int. J. Pharma. and Bio. Sciences, vol. 1(2), (2010), pp. 1-8.

[14] S. S. B. Ambomase, S. Tripathy, M. Tripathy and U. N. Dash, "Study on water-polymer interaction in the presence of aceclofenac at 298.15 K", E-Journal Chem., vol. 8(1), (2011), pp. 63-70.

[15] https://pubchem.ncbi.nlm.nih.gov/compound/Prasugrel_hydrochloride.

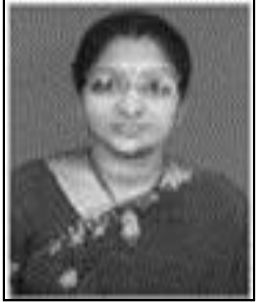

Lakshmi D, is currently pursuing Ph.D. in ultrasonics under the guidance of Prof. K. Samatha in Department of Physics, Andhra University, Visakhapatnam. She completed M.Sc. in Electronics from University of Hyderabad in the year 1998. She has been working as a Lecturer and has 19 years of teaching experience. At present she is also a Lecturer with Government Degree College, Tuni, Andhra Pradesh.

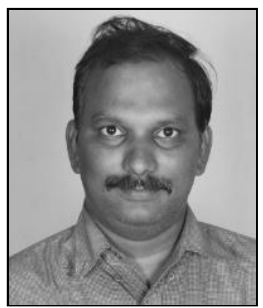

Venkata Ramana Ikkurthi, has been working as a Scientist in Department of Atomic Energy, since 1999. He finished M.Sc. in Physics from University of Hyderabad and obtained Ph.D. in Computational Physics from University of Griefswald, Germany. His areas of interest include Hydrodynamics, Fluid Dynamics, Material Modeling, High Performance Computing, Societal Applications of Computation, etc.

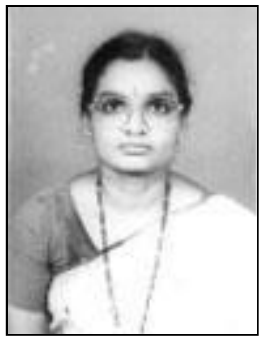

Prof. Samatha K, has been working as a Professor in Department of Physics, Andhra University, India. She finished M.Sc. in Solid State Physics and Ph.D. in Ultrasonics from Andhra University. Her areas of interest include Ultrasonics, Solid State Physics, etc. She was conferred best Thesis Award. She published more than 50 research articles in national and international journals. She guided about 8 M.Phil. and $10 \mathrm{Ph} . \mathrm{D}$. dissertations. 